\title{
The influence of physical ageing on the in-plane shear creep compliance of $5 \mathrm{HS}$ C/PPS
}

\author{
E.R. Pierik ${ }^{1}$ - W.J.B. Grouve ${ }^{1}$ M. van Drongelen ${ }^{1}$ (D) \\ R. Akkerman ${ }^{1}$
}

Received: 5 December 2018 / Accepted: 12 April 2019 / Published online: 30 April 2019

(C) The Author(s) 2019

\begin{abstract}
Thermoplastic polymer-matrix composites, such as carbon woven fabric reinforced poly(phenylene sulphide) (C/PPS), are increasingly used in the aircraft industry. Primary structural applications, however, are limited due to uncertainty concerning the longterm behaviour. Recent work indicated a progressive creep response over time, which would render these materials unusable for such applications. However, the effect of physical ageing was neglected, which is well known to alleviate the creep behaviour and hence physical ageing is rigorously included in this study on the long-term creep response of C/PPS. Shortterm tensile creep tests in the bias direction were performed at temperatures of 50, 60, 65, 70, 75 and $80^{\circ} \mathrm{C}$ to obtain a master curve using the time-temperature superposition principle. Ordinary horizontal shifting failed to produce a smooth curve and therefore three alternative approaches were used and compared. The physical ageing rate was, however, characterised with horizontal shifting only at $50^{\circ} \mathrm{C}$ and was implemented by means of the effective time theory (Struik, 1977) to correct the momentary master curves for the influence of physical ageing. The resulting predictions are more realistic and demonstrate that the structural changes in a material reduce the creep rate over time. Hence, the long-term creep compliance tends to increase asymptotically towards a finite value, in contrast to the unbounded momentary response.
\end{abstract}

Keywords Polymer-matrix composites (PMCs) - Thermoplastic resin · Creep · Physical ageing $\cdot 5 \mathrm{HS}$ C/PPS

\section{Introduction}

For some decades, composite materials in the form of fibre-reinforced polymer-matrix composites (PMCs) have been used to replace metal alloys in structural applications (Hastie 1991). The main reasons are the higher strength-to-weight and strength-to-stiffness ratios

M. van Drongelen

m.vandrongelen@utwente.nl

1 Faculty of Engineering Technology, Chair of Production Technology, University of Twente, Drienerlolaan 5, 7255 NB Enschede, The Netherlands 
Fig. 1 Long-term in-plane shear creep experiment according to the ASTM D3518/D3518M

standard (with a shorter specimen length of $180 \mathrm{~mm}$ ) on C/PPS material at $50^{\circ} \mathrm{C}$ and $\tau_{12}=5 \mathrm{MPa}$ with Kohlrausch-Williams-Watts (KWW) model fit as outlined by Motta Dias et al. (2016)

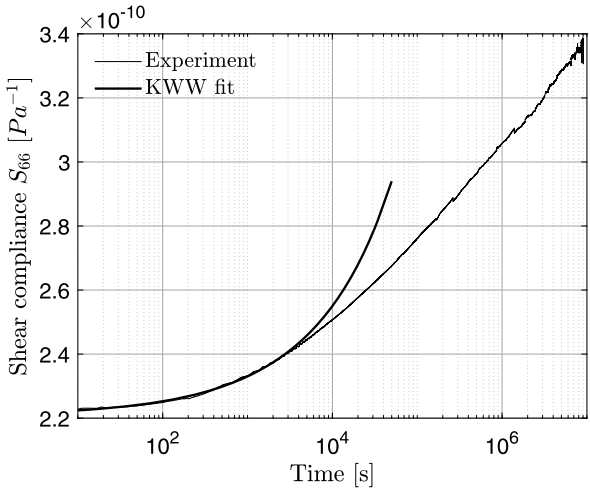

compared to metal alloys, which results in a weight reduction. The ongoing development is most evidently seen in the aircraft industry, where the use of composites has grown steadily over the past decades up to a point where PMCs now make up 53\% of the Airbus A350's structural weight (Airbus 2018). Civil aircraft are common to remain in service for 25 years, some even operate for nearly 50 years (Smith 2017). Clearly, the aircraft should be designed with this in mind, which requires insight in the long-term performance of the materials used. In the case of PMCs, off-fibre loading is primarily resisted by the matrix elasticity (Carnevale 2004), which is well known to be time-dependent (Barbero 2009). Further, the matrix material also affects the durability of the PMC (Brinson and Gates 1995). For example, physical ageing causes structural changes, which stiffens and embrittles the material, and thus affects the PMC's performance over time.

One of the popular thermoplastic PMCs is carbon woven fabric reinforced poly(phenylene sulphide) (C/PPS), of which the long-term performance is the subject of this study. Several researchers investigated C/PPS using bias extension or $\pm 45^{\circ}$ tensile tests to characterise the in-plane shear modulus under the influence of temperature or humidity changes or different fibre-matrix interfaces (e.g. Carnevale 2004; Albouy et al. 2012; Vieille and Taleb 2011; Vieille et al. 2012). Recently, Motta Dias et al. (2014, 2016) studied the effect of carbon fibre surface treatment on the long-term performance of C/PPS. In their study, specimens with treated and untreated fibres were subjected to isothermal creep experiments at various temperatures and physical ageing times. With the use of the time-temperature superposition principle (TTSP) and Struik's theory on physical ageing (Struik 1977), the obtained individual creep curves were shifted to construct a master curve, which spanned far longer times than the experimental window. The master curves of the two material systems were distinctively different, which indicates the influence of the fibre/matrix interface on the creep behaviour of C/PPS. The creep curves were modelled by means of the Kohlrausch or Kohlrausch-Williams-Watts (KWW) function, a stretched exponential function. The same approach was used in a research on neat PPS material carried out by Guo and Bradshaw (2007).

The cited research did not consider the ongoing effects of physical ageing on the creep behaviour of C/PPS over time, whereas the accompanying structural changes greatly influence the trend of a long-term creep response (Hastie 1991). This is illustrated in Fig. 1, in which a long-term creep experiment on C/PPS loaded in the exact same way as done by Motta Dias et al. (2016), spanning more than 100 days and performed in our lab, is shown together with a KWW function fit. The fit is based on data up to $3600 \mathrm{~s}$, since a larger time span reduces the goodness of fit with the first part of the creep measurement. The discrepancy between the KWW model and creep response grows over time up to a point at which 
the KWW model is not able to adequately represent the experimental creep data, which indicates that the procedure outlined is not able to fully characterise long-term creep behaviour. The effect of physical ageing on the creep response could be the missing link, which can be taken into account by means of the effective time theory (ETT) developed by Struik (1977). The ETT allows for correction of a non-aged (momentary) master curve to take into account the evolving effects of isothermal physical ageing. Several other researchers used the ETT in studies on physical ageing with satisfying results (Hastie 1991; Brinson and Gates 1995; Sullivan et al. 1993; Bradshaw and Brinson 1997). For example, Hastie (1991) concluded that without the ETT the obtained momentary master curve significantly overestimated the long-term in-plane shear creep compliance, whereas the compliance prediction with the ETT was only $7.3 \%$ lower than the measured one at the end of a long-term control experiment. To best of the authors' knowledge, such an application of Struik's ETT has not yet been performed on C/PPS.

Guo and Bradshaw (2009) did include the ETT in their study on the effects of nonisothermal ageing using an extension to the KAHR-model (Kovacs et al. 1979) on the creep response of unreinforced PPS material. The relation between PPS and C/PPS, however, is not that straightforward, due to the aforementioned fibre/matrix interphase effects (Motta Dias et al. 2016) and the difficulty in comparing stretch and shear data (Tschoegl et al. 2001). Furthermore, creep under influence of isothermal and non-isothermal ageing requires different approaches (Guo and Bradshaw 2009). Hence, the objective of this research is to correctly implement the TTSP with a correction for isothermal physical ageing using the procedure as outlined in Struik's ETT to predict the long-term in-plane shear creep compliance of carbon woven fabric reinforced PPS, which leads to a more realistic prediction of the actual creep behaviour.

\section{Background}

This section presents a brief overview of the tools and theories used in this research, such as the time-temperature equivalence and the physical ageing process.

\subsection{Time-temperature superposition principle}

Since a long-term prediction of the creep compliance is desired, there is need to accelerate experimental testing to reduce the required lab time. In 1941, Leaderman (1941) noted that creep curves at different temperatures do not change in shape, but shift in time. This concept, known as the time-temperature superposition principle (TTSP), can be used to construct a master curve based on several isothermal creep curves by altering the time scale with a shift factor, $a_{T}(T)$, to obtain a long-term prediction at a certain reference temperature (Griffith 1980).

\subsubsection{Thermo-rheologically simple vs. complex material}

Schwarzl and Staverman (1971) argued that if the isothermal creep response of a certain material is such that a change in temperature corresponds to a shift on the logarithmic time scale only, the material behaves as a thermo-rheologically simple material (TSM). This implies that the same sequence of molecular events occurs invariant of temperature, only the speed of these events is changed. Contrary to TSM behaviour, the sequence of molecular events 
may be altered or other molecular processes may be active under the influence of temperature in a thermo-rheologically complex material (TCM). Fesko and Tschoegl (1971) argued that all the retardation times in a TSM are affected by the same factor and thus the shift factor is independent of time, whereas the shift factor is also time dependent for a TCM. Gol'dman et al. (1978) mentioned that the equally affected retardation times for a TSM imply that the trend of a mechanical property is conserved on a log time scale with a change in temperature, which corresponds with the early notion of Leaderman (1941).

\subsubsection{Thermo-rheologically complex material shift}

One has to account for TCM behaviour if a horizontal shift on the logarithmic time scale is not sufficient to obtain a smooth master curve. A commonly applied approach is the addition of a vertical shift (Hastie 1991; Griffith 1980; Alwis and Burgoyne 2006; Amiri et al. 2016; Muliana et al. 2006). Although the horizontal scaling is based on a clear physical foundation, the same cannot be said about vertical scaling. Changes in density, thermal expansion or contraction, or humidity effects were mentioned in justification. Although linear viscoelasticity (LVE) is not a requirement for the TTSP (Griffith 1980), it seems, however, that the nonlinear range should be avoided (Hastie 1991). The application of both horizontal and vertical scaling is designated as graphical normalisation by Griffith (1980) and is often employed using a 2D error minimisation algorithm (e.g. Barbero 2010, 2009; Amiri et al. 2016). This procedure is often assumed to be valid in the case one obtains a smooth master curve. The easily implemented 2D error minimisation can, however, lead to an undesirable drift from the physical basis and errors in the prediction without attracting attention.

Griffith (1980) discussed several vertical shift models, from which the Tobolsky-Ferry method is most familiar (Brinson and Brinson 2015; Urzhumtsev 1974). This vertical factor is equal to $\rho T / \rho_{\text {ref }} T_{\text {ref }}$, which needs to be applied to the creep compliance curves measured at temperature $T$ with density $\rho$ prior to horizontal shifting to a reference temperature and density, $T_{\text {ref }}$ and $\rho_{\text {ref }}$, respectively. Urzhumtsev (1974) and McCrum and Morris (1966) provided another justification for the use of graphical normalisation. They showed that the vertical shift can be tweaked in such a way that the horizontal shift factor becomes independent of time, which indicates an equal shift for all the retardation times involved and thus TSM behaviour. A clear reasoning as regards the vertical shift factor was not provided, but it shows that a vertical scaling itself does not conflict with TSM behaviour.

In order to directly address the intrinsic property of TCM behaviour, i.e. the unequally affected retardation spectrum, Urzhumtsev (1974) and Gol'dman et al. (1977) investigated a temperature and time-dependent shift factor, $a_{T}(T, t)$. The proposed method is briefly presented in Appendix and boils down to the separation of $a_{T}(T, t)$ into a temperaturedependent part and a time-dependent part,

$$
\log a_{T}(T, t)=\log a_{T}\left(T, t_{0}\right)\left[1+\frac{\log a_{T}(T, \theta)}{\log a_{T}\left(T, t_{0}\right)}\right],
$$

where $t_{0}$ denotes a reference time, $T_{0}$ a reference temperature and $\theta=\log t-\log t_{0}$. The first term of the right-hand side only depends on temperature, whereas the last term still depends on both temperature and time. Hence, Urzhumtsev (1974) used a similarity function to obtain an expression invariant of temperature, which was approximated by a sum of exponentials. After integration, the obtained expression, $f(\theta)$, was used to approximate the 
last term of Eq. (1),

$$
\begin{aligned}
\log a_{T}(T, t) & =\log a_{T}\left(T, t_{0}\right)[1+f(\theta)] \\
& =\log a_{T}\left(T, t_{0}\right)\left[1+\frac{C}{n} \sum_{i=1}^{n}\left(1-e^{-\theta / b_{i}}\right)\right],
\end{aligned}
$$

in which the parameters $\left(C, b_{i}\right)$ were found empirically. Urzhumtsev (1974) checked the validity of the outlined procedure with a long-term control experiment on the creep behaviour of glass reinforced polyester, which resulted in a satisfactory agreement.

\subsection{Physical ageing}

A major progress on the characterisation of physical ageing in amorphous polymers has been achieved by Struik (1977). At temperatures below $T_{\mathrm{g}}$, the mobility in a material is too low to instantly reach thermodynamic equilibrium. Hence, volume, enthalpy and entropy are larger than in equilibrium. Over time, the difference in the specific volume and the occupied volume in the closest possible packing, denoted as free volume, reduces in a process to reach thermodynamic equilibrium. At the secondary transition temperature, $T_{\beta}$, segmental motion becomes impossible and hence the physical ageing process comes to a standstill, although more recently ageing below $T_{\beta}$ has been observed (e.g. Cerrada and McKenna 2000).

Struik (1977) argued that the segmental mobility decreases over time due to the reduction in free volume. As a consequence, retardation times increase and creep curves shift to longer times under influence of physical ageing. It was found that isothermal creep curves with different elapsed times (or ageing times), $t_{\mathrm{e}}$, can be superimposed with horizontal shifting on the logarithmic time scale. The superposition of creep curves with different $t_{\mathrm{e}}$ can be used to construct a master curve at a reference ageing time, as was done by for example Motta Dias et al. (2014, 2016). Struik (1977) found that a double-logarithmic representation of the horizontal acceleration or shift factors, $a_{\mathrm{e}}$, and the corresponding ageing times resulted in collinear points and hence a straight line could be drawn. The slope of this line is denoted as the ageing shift rate,

$$
\mu=-\frac{\mathrm{d} \log a_{\mathrm{e}}}{\mathrm{d} \log t_{\mathrm{e}}}
$$

which was used by Struik (1977) to characterise the physical ageing phenomenon for more than 40 materials. Struik (1977) noted that also in the case of semi-crystalline materials the amorphous regions are still subject to physical ageing and thus Eq. (3) remains valid.

Struik (1977) also performed measurements on non-isothermally aged material and concluded that previous ageing can be reversed if the ageing temperature is lower than the test temperature, since the processes that give rise to volume relaxation at a lower temperature are instantaneously met at higher temperatures. Non-isothermal ageing was more extensively studied by Kovacs et al. (1979), resulting in the KAHR-model, which was recently extended by Guo et al. (2009) to incorporate the relation with the mechanical response of a material that confirmed Struik's observations. Further, the effect of high stresses on physical ageing was investigated by Struik (1977). Experiments showed a rejuvenating effect, leading to the hypothesis that mechanical deformation results in an increase of the free volume, since deformations require segmental mobility and hence a certain amount of space, regardless of the nature of the applied stress. This hypothesis is under serious debate, according to Hutchinson (1995). McKenna (2003) experimentally proved that although a torsional deformation results in an instantaneous increase in volume, the disturbance relaxes towards the 
undeformed structural recovery curve after load removal in the case of temperatures close to $T_{\mathrm{g}}$ and deformations below the yield point. McKenna (2003) discussed that the apparent rejuvenation is more likely due to nonlinear viscoelastic memory effects than a change in the thermodynamic state. Anyhow, the notion of high stress influences on the physical ageing process remains important.

\subsection{Struik's effective time theory}

The notion that ageing results in structural changes is widely supported in the literature (e.g. Sullivan et al. 1993; Brinson and Gates 1995; Bradshaw and Brinson 1997; Gates and Feldman 1993; Hutchinson 1995). It was discussed that a reduction of segmental mobility in a quest for thermodynamic equilibrium results in a decrease in creep rate and impact strength, whereas the stiffness and yield stress increases over time. Hence, long-term creep cannot be considered without physical ageing taken into account (Hutchinson 1995). For that reason, Struik (1977) used the effective time theory (ETT) in his analysis. In the ETT, it is assumed that all retardation times are equally affected due to ageing with ageing shift rate $\mu$, as introduced in Eq. (3). Hence, the acceleration factor for a total ageing time of $t_{\mathrm{e}}+t$ with respect to a reference ageing time $t_{\mathrm{e}}$ can be characterised,

$$
a_{\mathrm{e}}\left(t_{\mathrm{e}}, t\right)=\left(\frac{t_{\mathrm{e}}}{t_{\mathrm{e}}+t}\right)^{\mu},
$$

which implies that the retardation times in a time interval $t$ to $t+\mathrm{d} t$ are $1 / a_{\mathrm{e}}$ times slower due to ageing with time $t$. Therefore, Struik (1977) argued that the time interval $\mathrm{d} t$ is $1 / a_{\mathrm{e}}$ times less eventful compared with a non-aged state. Hence, the interval $\mathrm{d} t$ can be related to an effective time interval, $\mathrm{d} \lambda$, in which no ageing takes place,

$$
\mathrm{d} \lambda=a_{\mathrm{e}}(t) \mathrm{d} t
$$

which can be integrated and combined with Eq. (4),

$$
\lambda(t)=\int_{0}^{t}\left(\frac{t_{\mathrm{e}}}{t_{\mathrm{e}}+\xi}\right)^{\mu} \mathrm{d} \xi,
$$

from which the effective time $\lambda$ as a function of $t$ can be obtained by evaluating the integral,

$$
\lambda(t)= \begin{cases}\frac{t_{e}}{1-\mu}\left[\left(1+\frac{t}{t_{e}}\right)^{1-\mu}-1\right] & \text { if } \mu \neq 1 \\ t_{e} \ln \left|1+t / t_{e}\right| & \text { if } \mu=1\end{cases}
$$

The effective time $\lambda$, in which no ageing takes places, corresponds to the momentary master curve creep time. Using Eq. (7), the momentary creep compliance on the $\lambda$-scale can be related with a real time $t$ to obtain a long-term prediction with a correction for physical ageing. Struik (1977) assumed the ageing shift rate to be equal to or smaller than unity in the derivation of Eq. (7), which is mathematically seen not required. Brinson and Gates (1995) discussed that, at least in the past, a $\mu$ equal to unity was considered as ideal. More recent experiments, however, revealed ageing shift rates exceeding unity, which are physically admissible (Brinson and Gates 1995). 


\section{Materials and methods}

The objective of this research is to predict the long-term creep compliance of C/PPS under influence of physical ageing. The physical ageing process can be quantified with the ageing shift rate, $\mu$, as introduced in Eq. (3). Afterwards, the TTSP can be used to construct a momentary master curve based on short-term creep measurements, which lack the influence of physical ageing over time. Hence, the momentary master curve needs to be corrected for physical ageing by means of Struik's effective time theory (Struik 1977), as shown in Eq. (7). This procedure will result in a similar curve as the long-term measurement illustrated in Fig. 1, but then spanning multiple years. Note that the long-term measurement was not used for further analysis, since a different experimental setup was used, the reference ageing time was uncertain and the statistical basis was too weak. The materials used and experiments conducted to gather the required data will be presented in this section.

\subsection{Materials}

The composite laminates under investigation were kindly supplied by ThermoPlastic composites Research Center, Enschede, the Netherlands, and were compression moulded out of eight semi-preg layers. The layers were stacked in $[(0,90)]_{4 s}$, with $(0,90)$ denoting one layer of fabric, to obtain an eight-ply-symmetric cross-ply laminate. The semi-preg was manufactured at Toray TCAC (formerly TenCate Advanced Composites), Nijverdal, the Netherlands, and consisted of 5-harness satin woven carbon fibres (Toray T300 3K) impregnated with poly(phenylene sulphide) (Ticona Fortron 0214), which has a glass transition and melting temperature of $90^{\circ} \mathrm{C}$ and $280^{\circ} \mathrm{C}$, respectively. Two equivalent laminates were compression moulded at $310^{\circ} \mathrm{C}$ and 10 bar, following the recommendations of the material supplier. $\mathrm{C}$-scans were made to check the quality of the laminates, which showed no voids or delaminations.

\subsubsection{Differential scanning calorimetry}

Differential Scanning Calorimetry (DSC) analyses were performed on a Netzsch Polyma DSC 214 with a nitrogen gas flow. The tests were carried out with heating and cooling rates of $10^{\circ} \mathrm{C} / \mathrm{min}$ up to $300^{\circ} \mathrm{C}$. Three samples were measured, namely:

1. As-received material.

2. Rejuvenated material. In this case the as-received material was subjected to a heat treatment at $120^{\circ} \mathrm{C}$ for 30 minutes.

3. Physical ageing (PA) tested material. In this case the DSC specimens were cut from a PA tested specimen, which was subjected to multiple creep-recovery tests after rejuvenation (see Sect. 3.3.2).

No clear difference was observed between the three samples regarding the degree of crystallinity, 30-32\% with a $\Delta H_{\mathrm{f}}^{0}$ of $150.4 \mathrm{~J} / \mathrm{g}$ (Spruiell and Janke 2004), the glass transition temperature, around $98-99^{\circ} \mathrm{C}$, and the melting peak with melting temperature around $283-$ $284^{\circ} \mathrm{C}$. From the above, it was assumed that no changes in morphology occurred during the experiments and the difference in material properties between both laminates was assumed to be negligible. 


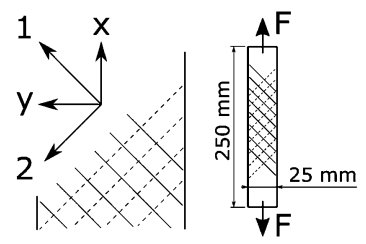

Fig. 2 Schematic of a $\pm 45^{\circ}$ fibre-orientated specimen with the principal material directions in the 1,2-frame. The $45^{\circ}$ rotated $x, y$-axes represent the loading coordinate frame, in which a force $F$ is applied in the $x$-direction during creep testing to characterise the shear compliance, as introduced in Eq. (8)

\subsubsection{Specimen preparation}

A water-cooled diamond blade saw was used to cut $\pm 45^{\circ}$ specimens from the laminates. Fig. 2 provides a schematic illustration of the $\pm 45^{\circ}$ specimens and the imposed loading direction. The principal material coordinates, denoted by the 1,2-frame, are aligned with the fibre directions. All specimens were dried for five days $(120 \mathrm{~h})$ in a vacuum oven (Binder VDL53) at $70^{\circ} \mathrm{C}$ and stored afterwards in a desiccator at room temperature to reduce possible effects of moisture on the creep response. The time at elevated temperature during the drying process also resulted in the required physical ageing of the specimens. The dimensions of all specimens were according to the ASTM D3518/D3518M standard (250 mm $\times$ $25 \mathrm{~mm}$ ). The thickness of the laminates was nominally $2.5 \mathrm{~mm}$ and the cross-sectional area of each specimen was calculated based on an average of three measurement points. Tabs were not required during creep testing.

\subsection{Test equipment}

The mechanical characterisation was performed on a Zwick Z100 tensile tester equipped with a $100 \mathrm{kN}$ load cell and a matching Grenco oven. A constant load was applied, which results in a varying stress over time due to cross-sectional deformation (Findley et al. 1976). The stresses and strains were small, however, and this effect can be safely assumed to be negligible. A thermocouple was attached to the specimens, consistently placed on the top part, to track the temperature during a test. The longitudinal and transversal strains were measured with Instron 2630-111 and 2620-602 extensometers.

\subsection{Experimental approach}

A tensile force applied in the $x$-direction, as illustrated in Fig. 2, was used to determine the composite's in-plane shear compliance,

$$
S_{66}=\frac{2 A\left(\varepsilon_{\mathrm{xx}}-\varepsilon_{\mathrm{yy}}\right)}{F},
$$

with $A$ the cross-sectional area, $F$ the applied tensile force and $\varepsilon_{\mathrm{xx}}$ and $\varepsilon_{\mathrm{yy}}$ the longitudinal and transversal strain, respectively. Equation (8) corresponds to the equation presented in the ASTM D3518/D3518M standard and valid under the assumption of small strains, as shown by Wisnom (1995). The shear stress in the principal material directions equals half the applied axial stress, $\tau_{12}=F / 2 A$ and the shear strain, $\gamma_{12}$, is defined as the longitudinal minus the transversal strain in the loading coordinate frame, $\varepsilon_{\mathrm{xx}}-\varepsilon_{\mathrm{yy}}$. The measured strains are time-dependent in the case of a creep experiment. Hence, the outcome of Eq. (8) becomes time-dependent, $S_{66}(t)$, which is denoted as the in-plane shear creep compliance (Griffith 1980). 
Fig. 3 Overview of the first part of the physical ageing test. Ageing starts after quenching the material at $t_{0}$ to below $T_{\mathrm{g}}$ and at an elapsed time of $t_{\mathrm{e}, 1}$ a constant load is applied for $0.1 t_{\mathrm{e}, 1}$, resulting in the first creep segment. At $t_{\mathrm{e}, 2}$, again a constant load is applied and the measured strain is corrected to account for the non-recovered strain $(\cdot-\cdot)$

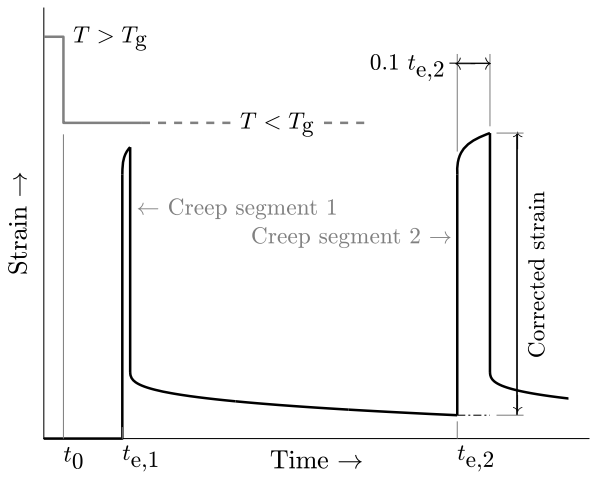

\subsubsection{Linear viscoelasticity tests}

Specimens were subjected to shear stresses ranging from 2.5 to $12.5 \mathrm{MPa}$ with steps of $2.5 \mathrm{MPa}$ at a temperature of $70^{\circ} \mathrm{C}$, in order to determine the linear viscoelastic (LVE) stress limit. The resulting creep compliance curves, based on the measured longitudinal and transversal strains, were compared with the reference curve measured at $2.5 \mathrm{MPa}$. The deviation in compliance is a measure of the nonlinearity, since the proportionality condition dictates that the compliance is invariant of the applied stress. The other requirement for LVE, Boltzmann's superposition principle, states that the creep response should be independent of previous loading. Hence, specimens were subjected to a second creep measurement and the deviation with the first creep response was determined. This was done for the 7.5 MPa shear stress loading condition.

The experiments were conducted with five specimens per condition, except for the 2.5 and 12.5 MPa loading condition, which were measured with three and two specimens, respectively. The specimens were first aged at $70^{\circ} \mathrm{C}$ for $120 \mathrm{~h}$ to exclude physical ageing effects during the creep tests. The specimens were settled down for at least 30 minutes after mounting to reach a steady state. Care was taken to maintain the applied force close to zero during this period.

\subsubsection{Physical ageing tests}

Physical ageing tests were carried out in order to determine the ageing shift rate, $\mu$, at $50^{\circ} \mathrm{C}$. The first part of such a test is illustrated in Fig. 3. Specimens were first separately rejuvenated at $120^{\circ} \mathrm{C}$ for 30 minutes in a Binder E28 oven, quenched to $50^{\circ} \mathrm{C}$ and immediately placed in the test setup. From that point in time, the physical ageing process starts, denoted with $t_{0}$ in Fig. 3. After a certain elapsed time, $t_{\mathrm{e}}$, a creep test at a constant shear stress was conducted followed by a recovery period, in which the load was held equal to zero, until a next creep segment was applied. The imposed shear stress was set equal to $5 \mathrm{MPa}$, based on the linear viscoelasticity tests. Elapsed times of 1, 3, 6, 12 and $24 \mathrm{~h}$ were used during a test and therefore one test comprises five creep responses with different ageing times. This procedure eliminates the need for repeated specimen installation. To exclude ageing effects during a creep segment, the so-called snapshot condition was obeyed, which limits the creep time to $0.1 t_{\mathrm{e}}$ (Struik 1977). The temperature was held constant at $50^{\circ} \mathrm{C}$ and the longitudinal and transversal strains were tracked over the full length of the experiment. A maximum temperature deviation of $0.15^{\circ} \mathrm{C}$ from the mean was observed. The measured strains were corrected for the non-recovered strain at the start of a new creep segment, 


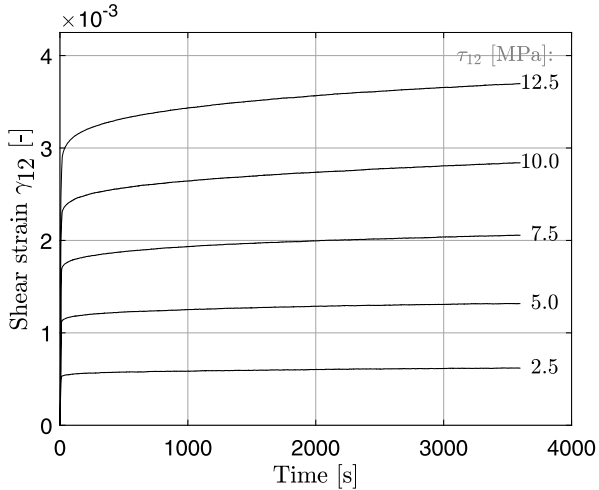

(a)

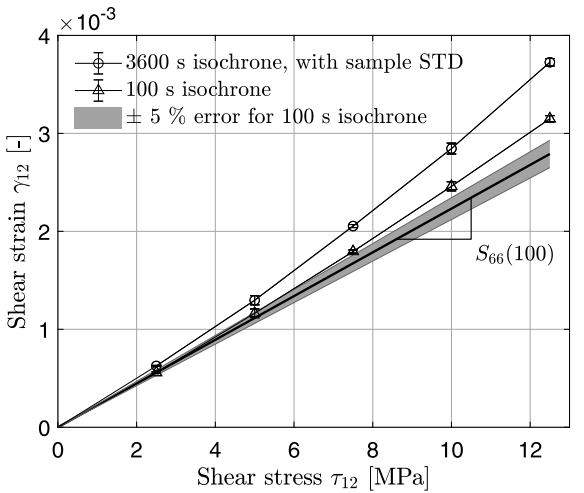

(b)

Fig. 4 Measured shear strain response with different stress levels in $\mathrm{MPa}$ at $70^{\circ} \mathrm{C}$ (a) and shear strain vs. shear stress isochrones at 100 and $3600 \mathrm{~s}$ for the proportionality check at $70^{\circ} \mathrm{C}(\mathrm{b})$, which should be straight for proportionality to hold. A 5\% error band in grey is added for the $100 \mathrm{~s}$ isochrone. The error bars represent the sample standard deviation (STD)

shown in Fig. 3 for creep segment 2, as advised by Struik (1977) and widely applied in other studies on physical ageing (e.g. Motta Dias et al. 2016; Gates and Feldman 1993; Sullivan et al. 1993). It was assumed that previous creep segments did not influence the outcome of subsequent creep segments, since stresses were within the linear viscoelastic range. This assumption was validated by control experiments.

\subsubsection{Creep tests}

Creep tests were conducted at temperatures of $50,60,65$ and $70^{\circ} \mathrm{C}$ for one hour to be able to construct a momentary master curve using the TTSP. Specimens were first aged at $70^{\circ} \mathrm{C}$ for $120 \mathrm{~h}$. Each condition was measured with the same five specimens, starting from the lowest test temperature. In addition, creep tests were performed at 75 and $80^{\circ} \mathrm{C}$ to extend the time span of the master curve, for which specimens were aged for $120 \mathrm{~h}$ at their respective test temperatures instead of $70^{\circ} \mathrm{C}$ to avoid partial or complete rejuvenation of the specimens during the creep experiments. The proportionality condition was evaluated at $80^{\circ} \mathrm{C}$ to ensure linear viscoelasticity before the actual creep experiments took place at 75 and $80^{\circ} \mathrm{C}$.

\section{Results}

Here, the linear viscoelastic stress limit and ageing shift rate will be determined based on experimental data. The measured isothermal creep curves will be used to construct momentary master curves. This section concludes with a correction of the master curves to take into account the effect of physical ageing over time.

\subsection{Linear viscoelasticity}

Figure $4 \mathrm{a}$ visualises the effect of the shear stress level on the measured creep response at $70^{\circ} \mathrm{C}$. The shear strain was evaluated at 100 and $3600 \mathrm{~s}$ and the resulting isochrones with the corresponding shear stress, $\tau_{12}$, are shown in Fig. $4 \mathrm{~b}$. The isochrones should be linear 


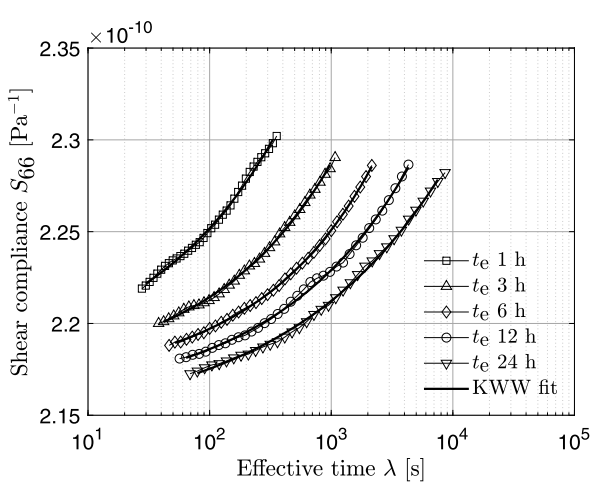

(a)

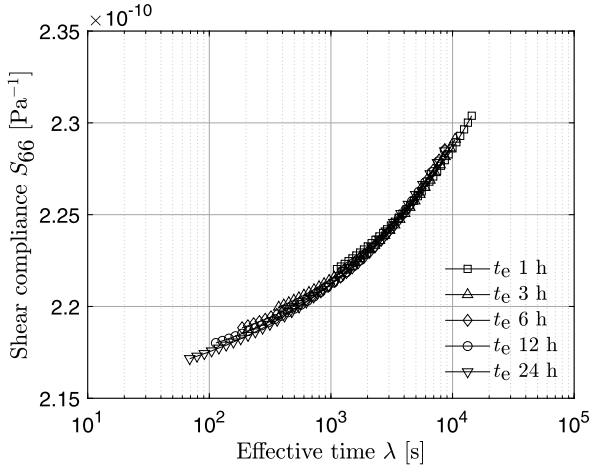

(b)

Fig. 5 Creep curves of one specimen measured at $50^{\circ} \mathrm{C}$ with different elapsed times, $t_{\mathrm{e}}$, and fitted with the KWW function (a), which were horizontally shifted (b) to obtain appropriate shift factors

for proportionality to hold, which is not the case as illustrated by the deviation of the $100 \mathrm{~s}$ isochrone from the thick line in Fig. 4b, with a 5\% error band in grey. Straight isochrones indicate an in-plane shear creep compliance invariant of shear stress, shown by the slope triangle. Hence, the measured strains were used to compute the creep compliance by means of Eq. (8) and the deviation in compliance at 100, 1800 and $3600 \mathrm{~s}$ was computed with the $2.5 \mathrm{MPa}$ compliance values as a reference. A maximum deviation in compliance of $5 \%$ was considered to be acceptable, which resulted in a shear stress limit of $5 \mathrm{MPa}$.

The specimens which were already subjected to a creep experiment at $7.5 \mathrm{MPa}$, were measured again at the same stress level and temperature in order to check whether Boltzmann's superposition principle holds. It was observed that the creep response of the second measurement was in good agreement with the first measurement (within 5\%). Hence, the LVE stress limit was dictated by the proportionality condition. Therefore, the LVE shear stress limit was set equal to $5 \mathrm{MPa}$, which was used in further experiments. In addition, proportionality was also checked at $80^{\circ} \mathrm{C}$, confirming the conclusion above. The shear stress limit chosen was in agreement with the ones used in the studies of Motta Dias et al. on $\pm 45^{\circ}$ C/PPS (Motta Dias et al. 2014; Motta Dias et al. 2016).

\subsection{Physical ageing}

Five creep segments were measured during each physical ageing tests corresponding to the different ageing times, $t_{\mathrm{e}}$, of 1, 3, 6, 12 and $24 \mathrm{~h}$. A strain correction was applied on each segment to ensure a zero strain value right before load application. The measured strains were used to compute the in-plane shear creep compliance by means of Eq. (8). The result of one of the measured specimens is shown in Fig. 5a. Distinct, non-coinciding, creep curves arise as a function of the ageing time, which illustrates the effect of the structural changes in the material over time. The creep rate decreases significantly due to a reduction in free volume, which hinders the segmental mobility. The creep compliance curves are represented as a function of the effective time, $\lambda$, which means that no ageing was assumed to take place during each creep segment. The momentary creep curves were fitted with the Kohlrausch or Kohlrausch-Williams-Watts (KWW) function,

$$
S_{66}(t)=S_{0} e^{(t / \tau)^{\beta}},
$$


Fig. 6 Negative logarithmic ageing shift factors, $-\log a_{\mathrm{e}}$, for a reference ageing time of $24 \mathrm{~h}$ shown with ageing time, $t_{\mathrm{e}}$, on a logarithmic scale based on isothermal experiments $\left(50^{\circ} \mathrm{C}\right)$. The slope of the line in the double-logarithmic plot corresponds to the ageing shift rate, $\mu$, as introduced in Eq. (3)

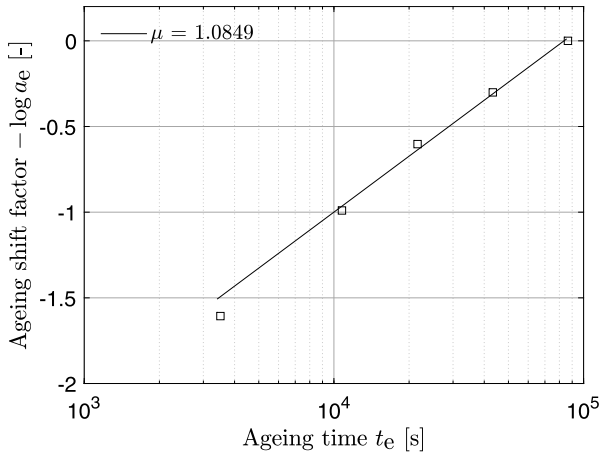

with $S_{0}$ the initial compliance, $\tau$ a parameter proportional to the retardation time and $\beta$ a parameter inversely related to the width of the distribution of retardation times (Flory and McKenna 2004).

The fitted creep curves were then shifted horizontally to obtain horizontal ageing shift factors, $a_{\mathrm{e}}$, which correspond to a certain $t_{\mathrm{e}}$. The horizontal shift to a reference creep curve of $24 \mathrm{~h}$ ageing was carried out by means of a least squares error minimisation on the difference in compliance, leading to the results shown in Fig. 5b. The creep curves coincide to one master curve, which implies that all retardation times are equally affected by physical ageing and the accompanying reduction in free volume. A double-logarithmic plot of $a_{\mathrm{e}}$ versus $t_{\mathrm{e}}$ for the same specimen is shown in Fig. 6. The collinearity of the points together with the smooth master curve indicate that C/PPS behaves conform the physical ageing theory outlined by Struik (1977) (see Sect. 2.2). The slope of a straight line through the collinear points corresponds to the ageing shift rate, $\mu$, as introduced in Eq. (3). The ageing shift rate was calculated for each specimen and the average of four specimens was computed. In the case all data points were used for the linear fit, $\mu_{\text {avg }}$ equalled 1.090 with a (sample) coefficient of variation $(\mathrm{CoV})$ of $6.55 \%$. If the $1 \mathrm{~h}$ data was excluded, as shown in Fig. $6, \mu_{\mathrm{avg}}$ equalled 1.075 and the $\mathrm{CoV}$ reduced to $1.63 \%$. The latter value was used in the upcoming analyses, as it was considered to be more reliable.

The sequential loading and unloading during the physical ageing (PA) tests could influence the creep response in the following creep segments, affecting the physical ageing analysis. Therefore, control experiments were conducted to check if the previous loading and unloading cycles ( $t_{\mathrm{e}}$ of 1, 3, 6 and $12 \mathrm{~h}$ ) influenced the strain response of the last creep segment $\left(t_{\mathrm{e}}\right.$ of $\left.24 \mathrm{~h}\right)$. Two PA tested specimens were first rejuvenated and subjected to a creep measurement after an ageing time of $24 \mathrm{~h}$. The creep response was compared with the earlier obtained response in the PA test of the same specimen. It was observed that the deviations in compliance were only small, within $1.2 \%$. Thus, the sequential loading and unloading during the physical ageing test did not influence the subsequent creep response.

\subsection{Momentary master curves}

The average shear creep compliance curves at different temperatures versus the effective time are shown in Fig. 7a, which visualises an increase in creep rate with temperature. This illustrates the higher mobility and shorter retardation times at elevated temperature, as expected from the kinetic rate theory (Leaderman 1941). The sample standard deviation at $1800 \mathrm{~s}$ is added in Fig. 7a, which is representative for the whole time range. Up to and including the $70^{\circ} \mathrm{C}$ data, the coefficient of variation was lower than $1.3 \%$. The variation in 


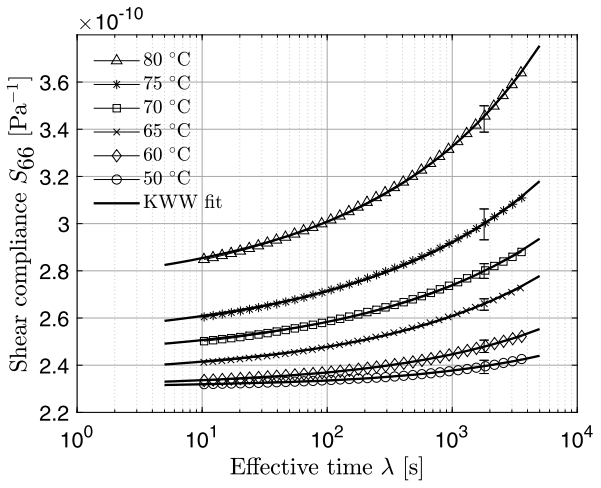

(a)

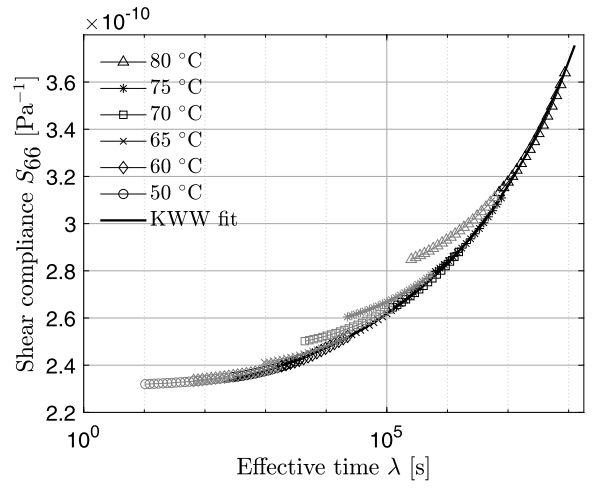

(b)

Fig. 7 Average creep curves at various temperatures with KWW fit (a), which were shifted using only horizontal shift factors to obtain a master curve (b), denoted as HS master curve, with excluded data in grey $\left(T_{\text {ref }}=50^{\circ} \mathrm{C}\right.$ and $\left.t_{\mathrm{e}}=120 \mathrm{~h}\right)$

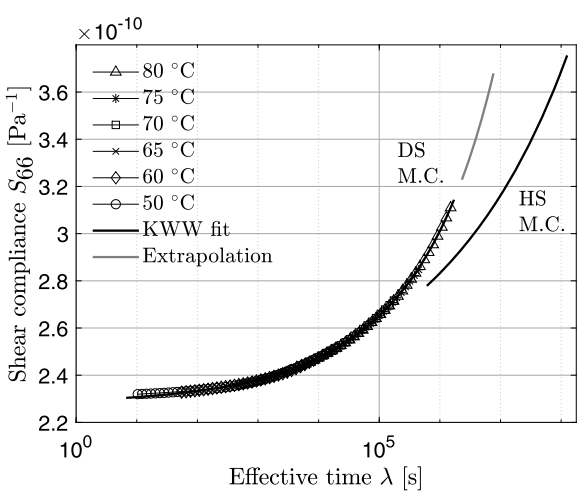

(a)

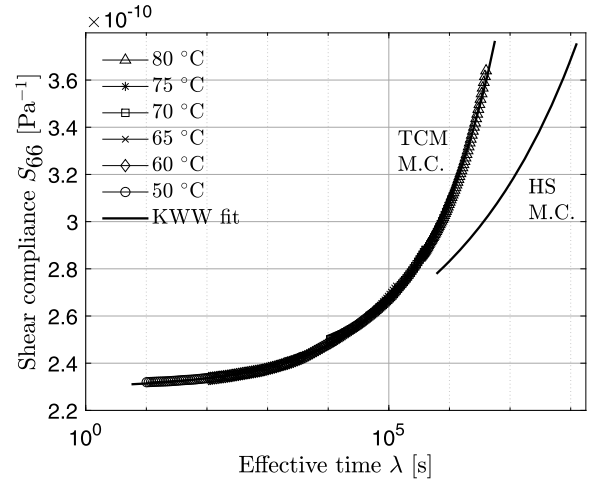

(b)

Fig. 8 A double (horizontal and vertical) shift (a) and a TCM shift (b) of the of the creep curves shown in Fig. 7a, denoted as DS master curve, with an extrapolation based on a KWW fit in grey, and TCM master curve, respectively $\left(T_{\text {ref }}=50^{\circ} \mathrm{C}\right.$ and $\left.t_{\mathrm{e}}=120 \mathrm{~h}\right)$. Part of the $\mathrm{HS}$ master curve is added for comparison

the 75 and $80^{\circ} \mathrm{C}$ data remained within $2.3 \%$. The average creep compliance curves were fitted with the KWW function (9) and the corresponding parameters are provided in Table 1, which shows that the parameter proportional to the retardation time, $\tau$, indeed reduces with increasing temperature.

The data points were then shifted to obtain momentary master curves at a reference temperature of $50^{\circ} \mathrm{C}$ with an ageing time of $120 \mathrm{~h}$ using TTSP. Three different approaches were carried out, namely only a horizontal shift with omission of start-up data, a double (horizontal and vertical) shift and a TCM shift. The horizontally shifted (HS) master curve was obtained using the same error minimisation algorithm as used with the physical ageing procedure and is shown in Fig. $7 \mathrm{~b}$. Note that the first $300 \mathrm{~s}$ were omitted in this approach to smoothen the master curve, since otherwise no reasonably smooth master curve could be obtained. The excluded data is added in grey symbols in Fig. 7b. A direct shift of the 75 and $80^{\circ} \mathrm{C}$ data could not be executed due to a lack of overlap, which was solved by implementing 


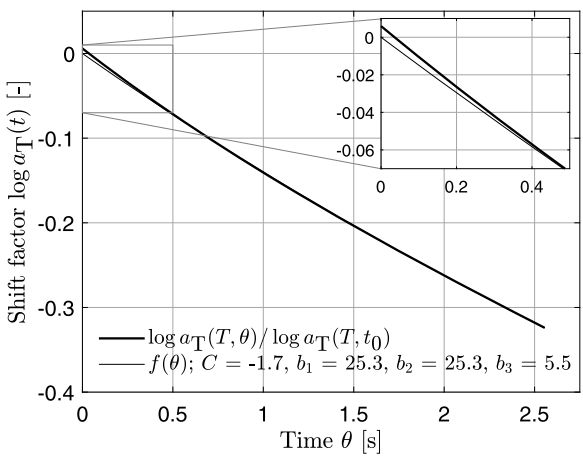

(a)

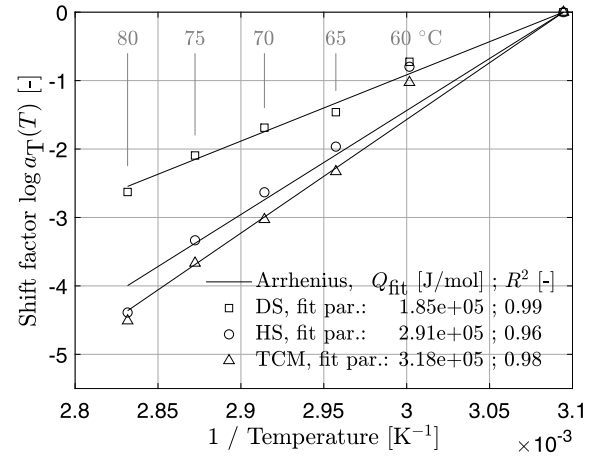

(b)

Fig. 9 Time-dependent part of the TCM shift (thick line) with approximation $f\left(\theta=\log t-\log t_{0}\right)$ of the shift between 50 and $60^{\circ} \mathrm{C}$ creep curves (a) and the horizontal shift factors of the horizontally shifted (HS), double-shifted (DS) and TCM master curve (b) fitted with the Arrhenius equation (10) $\left(T_{\text {ref }}=323.15 \mathrm{~K}\right)$

Fig. 10 Correction of the momentary master curves for the influence of physical ageing on the long-term performance of $\pm 45^{\circ} \mathrm{C} / \mathrm{PPS}$ with $T_{\text {ref }}=50^{\circ} \mathrm{C}$ and $t_{\mathrm{e}}=120 \mathrm{~h}$

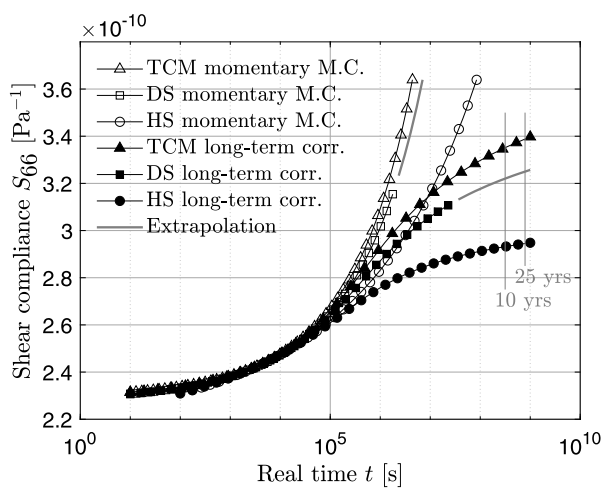

a KWW fit on the lower temperature data followed by a shift of the 75 and $80^{\circ} \mathrm{C}$ data points onto this KWW fit.

The momentary master curve of the second approach was based on double (horizontal and vertical) shifting (DS). The error minimisation routine was extended by an additional parameter, $b_{T}$, and the result is shown in Fig. 8a. This time, there was no need to neglect the start-up data in order to obtain a smooth master curve. The used vertical shift factors, $b_{T}$, were $0.997,0.97,0.94,0.92$ and 0.86 for the 60 up to $80^{\circ} \mathrm{C}$ data, respectively. Hence, the creep curves were shifted downwards and a monotonic trend with temperature was observed for the vertical shift factors. A KWW fit was used to extrapolate the master curve, as shown by the grey line in Fig. 8a, since the application of vertical shifting cropped the data. A part of the KWW fitted HS master curve is added for comparison. Both master curves are distinctly different, although based on the same experimental data.

The third approach made use of Eq. (2) with three terms in the series expansion. The parameters of the time-dependent part, $\left(C, b_{i}\right)$, were computed using the shift between the 50 and $60^{\circ} \mathrm{C} \mathrm{KWW} \mathrm{fitted} \mathrm{creep} \mathrm{curves.} \mathrm{The} \mathrm{temperature} \mathrm{and} \mathrm{time-dependent} \mathrm{shift,} \log a_{T}(T, t)$, was calculated using the horizontal time distance between equal compliance values on both curves. The temperature-dependent part, $\log a_{T}\left(T, t_{0}\right)$, should be constant with time, which was used to determine $C$ and $b_{i}$ by means of error minimisation. The base time, $t_{0}$, was set 
equal to the first data point of the creep curves (10 s). The obtained approximation of the time-dependent part, $f(\theta)$, is compared with the computed one in Fig. 9a. This approximation was used to construct the shift of the other datasets, in which the temperature-dependent shift was found by error minimisation as if it were an ordinary horizontal shift. The obtained TCM master curve is shown in Fig. 8b, which is quite smooth and shows a fair resemblance with the DS master curve regarding its shape. Note that the DS master curve was cropped due to vertical shifting and hence extrapolation was required, whereas this was unnecessary for the TCM shift.

The horizontal shift factors, $a_{T}$, for all three approaches are presented in Fig. 9b. The creep behaviour in the glassy range is often characterised by means of the kinetic rate theory (Griffith 1980). Hence, it is common practise to fit horizontal shift factors with the Arrhenius equation,

$$
\ln \left(a_{T}\right)=\frac{Q}{R}\left(\frac{1}{T}-\frac{1}{T_{\text {ref }}}\right),
$$

with temperature $T$ and reference temperature $T_{\text {ref }}$ both in Kelvin, $Q$ the activation energy (per mole) and $R$ the gas constant. In Fig. 9b, use has been made of the Arrhenius equation (10) to fit the horizontal shift factors. The corresponding activation energies are shown in the legend together with the $R^{2}$-values. The activation energies were quite different, although the three master curves were based on the same creep curves with a certain secondary bond strength. The conformity with the Arrhenius equation (10) is, however, more relevant, as it shows the conformity with theory. The DS shift factors did follow the Arrhenius equation quite well as indicated by an $R^{2}$-value of 0.99 , whereas the HS and TCM shifts were too small at $60^{\circ} \mathrm{C}$ (as much as 43 and $33 \%$ off on log scale in relation to the Arrhenius fit, respectively). Furthermore, the $\mathrm{HS}$ shift factor at $80^{\circ} \mathrm{C}$ was a bit too high compared to the fit $(9.8 \%)$.

\subsection{Correction for physical ageing}

Figure 10 shows the three obtained KWW fitted momentary master curves. The curves need to be corrected to incorporate the influence of physical ageing in order to obtain a prediction of the true long-term performance. Struik's effective time theory (Struik 1977) was used to relate the creep compliance values on the effective time scale, $\lambda$, to a real time scale, $t$, by means of Eq. (7), in which the earlier determined average physical ageing shift rate, $\mu_{\text {avg }}$, was used (see Sect. 4.2). The reference ageing time was set equal to $120 \mathrm{~h}$, as the momentary creep response was based on $120 \mathrm{~h}$ aged specimens. The result of this analysis is shown in Fig. 10 for all three approaches. The structural changes in a material reduce the creep rate and hence the long-term creep compliance seems to increase asymptotically towards a finite compliance value over time. With this, the main goal of this research was achieved: the effect of the ongoing structural changes in C/PPS was incorporated in a long-term prediction of the in-plane shear creep compliance.

\section{Discussion}

In this section the obtained results will be related to the available literature and the validity of the experimental data will be discussed. Further, the significance of this research will be interpreted and discussed in a wider context, for which several theories pass in review. 
Table 1 KWW fit parameters for average creep curves at different temperatures (Fig. 7a) and for the HS, DS and TCM master curves (Figs. 7b, 8a and 8b)

\begin{tabular}{lllllllllll}
\hline & & $50^{\circ} \mathrm{C}$ & $60^{\circ} \mathrm{C}$ & $65^{\circ} \mathrm{C}$ & $70^{\circ} \mathrm{C}$ & $75^{\circ} \mathrm{C}$ & $80^{\circ} \mathrm{C}$ & HS & DS & TCM \\
\hline$S_{0}$ & {$\left[\times 10^{-10} \mathrm{~Pa}^{-1}\right]$} & 2.3077 & 2.3090 & 2.3517 & 2.4233 & 2.4880 & 2.6932 & 2.1475 & 2.2766 & 2.2903 \\
$\tau$ & {$\left[\times 10^{6} \mathrm{~s}\right]$} & 7.5977 & 3.7808 & 2.1407 & 1.8007 & 1.0081 & 0.2530 & 6898.6 & 122.58 & 61.152 \\
$\beta$ & {$[-]$} & 0.3940 & 0.3465 & 0.2958 & 0.2804 & 0.2648 & 0.2809 & 0.1453 & 0.2648 & 0.2920 \\
\hline
\end{tabular}

\subsection{Physical ageing}

The ageing shift rate of C/PPS in the bias direction was also addressed in a study of Motta Dias et al. (2016), in which an average rate of around 1.1 was found at $50^{\circ} \mathrm{C}$. Strain corrections were made on each individual creep segment. In a study of Guo and Bradshaw (2007), neat PPS material was investigated. No notion of strain correction was made and an average value of 1.137 was found at $57^{\circ} \mathrm{C}$. The ageing shift rate obtained in this work, 1.075 at $50^{\circ} \mathrm{C}$ with omission of the $1 \mathrm{~h}$ data, is similar to the aforementioned literature values.

The omission of the first creep response, $t_{\mathrm{e}}$ of $1 \mathrm{~h}$, in the determination of the ageing shift rate resulted in a significantly lower coefficient of variation. Mechanical conditioning could serve as an explanation for this observation and for the required strain correction after each creep segment. Namely, Leaderman (1941) found that the recovery after a first creep measurement on filamentous materials did not approach zero, whereas the recovery after a second measurement did. The non-recoverable deformation after a first creep measurement was referred to as mechanical conditioning, which could be due to orientation of the amorphous and crystalline regions in the material. Zapas and Crissman (1984) found the same mechanical conditioning behaviour in the case of polyethylene material. The additional nonrecoverable creep deformation accompanying the first creep segment increases the response, whereas this additional creep is expected to be lower during the sequential creep segments. Another clarification for the larger variation with $1 \mathrm{~h}$ data taken into account might be found in the first minutes of a PA test due to differences in the exact initial temperature, heating rate and temperature overshoot, which affects the effective ageing time. This distortion has the largest influence on the first measurement, due to the relatively short ageing time. As a consequence of the short ageing time of $1 \mathrm{~h}$ in combination with the snapshot condition, the accompanying creep time is also short. Therefore, the resulting curve has only a small curvature, which results in a less pronounced shift.

\subsection{Momentary master curves}

The horizontally shifted master curve without the omission of start-up data, as shown in Fig. 7b, was not smooth. Hence, one of the most important requirements for the TTSP to succeed, was violated. Three approaches were attempted to resolve this, namely a horizontal shift with omission of data (HS), a double (horizontal and vertical) shift (DS) and a thermo-rheologically complex material (TCM) shift. The resulting momentary master curves are shown in Fig. 11a. First, a relation will be drawn with the horizontal shift carried out by Motta Dias et al. on C/PPS (Motta Dias et al. 2016). The section concludes with a thorough discussion on the three obtained master curves and the observed TCM behaviour. 


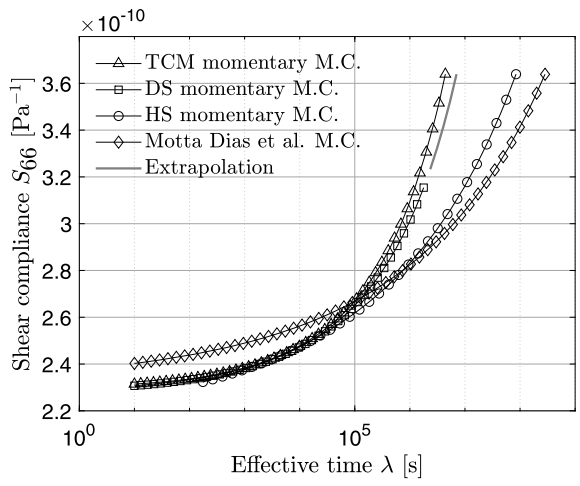

(a)

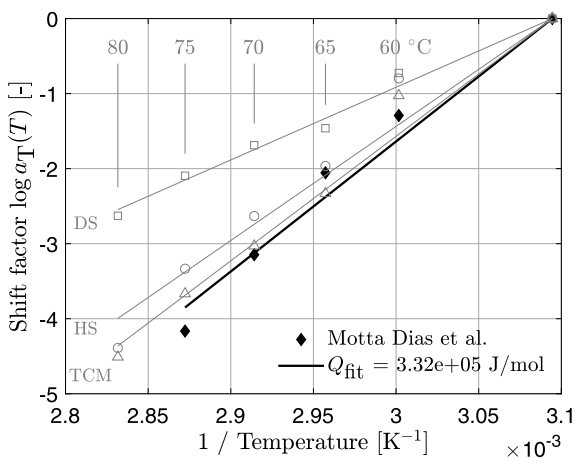

(b)

Fig. 11 Momentary master curve of Motta Dias et al. (2016) compared with the three master curves obtained in this research (a) and the accompanying horizontal shift factors (b) with $T_{\text {ref }}=50^{\circ} \mathrm{C}$ and $t_{\mathrm{e}}=120 \mathrm{~h}$

\subsubsection{Horizontal shift factors}

Motta Dias et al. (2016) performed measurements on two C/PPS material systems. One of them, the surface modified (SM) carbon fibre, was the same material as used in this research (Luinge 2018). The master curve was constructed by horizontal shifting only, without omission of start-up data, with a reference temperature and ageing time of $40^{\circ} \mathrm{C}$ and $96 \mathrm{~h}$. The curve is shifted to $50^{\circ} \mathrm{C}$ and $120 \mathrm{~h}$ by means of the provided shift factors and ageing shift rate in order to compare the momentary master curve to the ones constructed in this work, as shown in Fig. 11a. The obtained master curve has a higher initial compliance and a lower creep rate, which could be due to differences in material properties, specimen preparation or the setup used. The horizontal shift factors can be found in Fig. 11b, which result in an activation energy of $3.32 \mathrm{e} 5 \mathrm{~J} / \mathrm{mol}$, which is a bit higher than the ones obtained in this research (see legend of Fig. 9b). Guo and Bradshaw (2007) characterised the creep behaviour of neat PPS material using only horizontal shifting, from which an activation energy of $2.75 \mathrm{e} 5 \mathrm{~J} / \mathrm{mol}$ can be obtained.

The shift factors of Motta Dias et al. (2016) at 60 en $65^{\circ} \mathrm{C}$ are a bit too low $(20$ and $14 \%$ off, respectively), whereas the shift at $75^{\circ} \mathrm{C}$ is larger than the Arrhenius fit $(8.2 \%)$. Therefore, Motta Dias et al. (2016) used a log-polynomial function to fit the shift factors, instead of the Arrhenius equation (10). More or less the same deviating behaviour with respect to the Arrhenius equation was observed in the case of the HS shift conducted in this research, which also tends to follow a curve that flattens at lower temperature. Struik (1977) argued that if the temperature is sufficiently below $T_{\mathrm{g}}$, relaxation times tend to vary linearly with temperature instead of exponentially. More data points are required to verify this hypothesis in the case of the material and loading condition considered in this study. Although the transition from exponentially towards linearly dependent retardation times could be an explanation for the poor conformity with the Arrhenius equation, it does not diminish the suspicion about the validity of the obtained HS master curve, as the obtained curve was not really smooth either (see Fig. 7b).

\subsubsection{Master curve construction}

The HS master curve could only be constructed in the case that start-up data was omitted. The validity of this approach will be discussed here. In a study conducted by Alwis and 
Burgoyne (2006) on creep in aramid fibre composite, it was discussed that the rapid changes in the creep gradient in the beginning of the creep measurement can be neglected in order to obtain steady state creep curves. This resulted in better matching creep curves and hence a reasonably smooth master curve. Hung and Wu (2018) used the stepped isostress method and mentioned that load application and creep history had the largest influence in the primary creep region and hence primary creep was eliminated to obtain a smooth master curve. Lee and Knauss (2000) argued that a finite ramp time instead of an ideal step results in a different viscoelastic response, which converges over time. This influence is negligible after ten times the ramp time, according to their model. Flory and McKenna (2004) compared the model of Lee and Knauss with the Zapas-Craft model and found that, especially for materials with low relaxation times, the Lee and Knauss assumption of ten times the ramp time results in significant errors. In the case of materials with longer relaxation times this 'factor of ten rule' results in a reasonable approximation. It should be noted that, to the authors' knowledge, this procedure is mostly seen in stress relaxation or nano-indentation measurements and not so frequently in creep testing. However, the proposed load disturbance could be a justification for the omission of start-up data.

The DS master curve obtained in this research was based on the whole creep curves, without omission of start-up data, and resulted in a smooth master curve (Fig. 8a) with shift factors that followed the Arrhenius equation to a reasonable extent (Fig. 9b). In the case that start-up data corresponding to ten times the ramp time was excluded, as proposed by Lee and Knauss (2000), no significant changes occurred, only a slight deflection towards the TCM master curve was observed.

The vertical scaling could be modelled with Tobolsky-Ferry method (see Sect. 2.1.2). With the assumption that density effects are negligible (Brinson and Brinson 2015), the application of the Tobolsky-Ferry method increases the deviation in initial compliances and hence worsens the smoothness of the master curve, as also mentioned by Griffith (1980). Urzhumtsev (1974) discussed that the Tobolsky-Ferry factor reflects changes in entropy as it is based on the theory of rubber elasticity, whereas in the case of polymers with a stiffer macromolecule, the deformation is dominated by energy effects and therefore results in a decreasing modulus with temperature. Brinson and Brinson (2015) supported this statement by noting that deformations in the glassy state include stretching and shortening of bond distances and angles, whereas the rubbery state is typified by rotations about bond angles. Bradshaw and Brinson (1997) discussed in their study on time-ageing time superposition that no clear theory exists for vertical scaling, only that it acts on the initial compliance of the creep curves. Table 1 lists the KWW fit parameters for the isothermal creep curves. The parameter associated with the initial compliance, $S_{0}$, increases with temperature. Application of the obtained vertical shift factors (see Sect. 4.3) indeed equalises the initial compliances. This method shows resemblance with the one used by Kê (1947) on creep in aluminium, who divided the total creep response by the instantaneous deflection prior to horizontal shifting. Smooth master curves can be obtained in the glassy region in the case the temperature dependency of the instantaneous deflection is taken into account, according to Griffith (1980).

The TCM master curve was obtained by means of the shift proposed by Urzhumtsev (1974). This method provides a more fundamental approach to modelling the TCM behaviour, as it takes changes in the retardation spectrum explicitly into account. It was observed that the calculation of $f(\theta)$ was especially difficult at lower values of $\theta$, as shown by the inset in Fig. 9a. Perhaps another function fit is able to provide a better approximation, but it also suggests that especially the start-up phase deviates from TSM behaviour. Possibly, one of the mentioned disturbances, i.e. large creep gradient changes (Alwis and Burgoyne 
2006) or a non-ideal step (Lee and Knauss 2000), are responsible for this behaviour. Start-up data was, however, not excluded, since the TCM procedure projected the shape of the shifted creep curve onto the shape of the reference creep curve at longer creep times and therefore these possible effects were taken into account in the shift. The obtained master curve was smooth (Fig. 8b) and the conformity of the shift factors with the Arrhenius equation (10) was reasonable (Fig. 9b).

\subsubsection{Thermo-rheologically complex behaviour}

Two out of three master curves constructed in this research are based on the assumption of TCM behaviour. The complex behaviour could be due to the interaction between the amorphous and crystalline part, according to Lai and Bakker (1995). However, Guo and Bradshaw (2007) obtained a smooth master curve with horizontal shifting alone on PPS material, albeit with unknown crystalline morphology. The interaction with fibres could be another reason for the TCM behaviour observed in the C/PPS considered in this work. Nonetheless, Motta Dias et al. (2014, 2016) assumed TSM behaviour in their studies on C/PPS.

Although the TCM behaviour is remarkably in the TTSP, the creep behaviour in the physical ageing analysis is obviously thermo-rheologically simple, as shown by the successful horizontal shift of the creep curves with different ageing times (Fig. 5b) and the collinearity of the used shift factors (Fig. 6). The resemblance between the TTSP and the physical ageing procedure is clear: both use horizontal shifting to match individual creep curves. Hence, the observation of both TSM and TCM behaviour seems contradicting. Ageing time is the driving factor for a difference in mobility in the physical ageing analysis. Ageing results in a lower free volume, which in its turn hinders mobility, as discussed by Struik (1977). Thus, the creep response strongly depends on the available free volume. On the contrary, temperature is the driving factor in the TTSP analysis. Since the experiments were performed below $T_{\mathrm{g}}$, the free volume remains almost constant with temperature and the mobility is dominated by thermal activation (Struik 1977). Hence, the analyses are based on different mechanisms, which implies that one does not preclude the other. Struik (1977) noted that above $T_{\mathrm{g}}$ the equilibrium free volume is temperature-dependent, whereas it becomes time-dependent below $T_{\mathrm{g}}$. Therefore, if the shape of the creep curves above $T_{\mathrm{g}}$ is invariant under temperature, the same should be observed below $T_{\mathrm{g}}$ with a change in ageing time, corresponding to TSM behaviour.

\subsection{Correction for physical ageing}

The physical ageing correction of the obtained master curves, shown in Fig. 10, illustrates the strong effect of the continuous structural changes in a material, which leads to stiffening and hence reduces the creep rate over time. A clear difference in the trend of the curves is observed between the momentary response, in which the compliance response seems to be unbounded, and the curve corrected for physical ageing, in which the compliance tends to increase asymptotically towards a finite value. Hence, the momentary prediction greatly overestimates the long-term compliance. Although in practise a clever design and proper laminate lay-up will be used to avoid an in-plane shear load case, the overestimation of the creep response will lead to overdimensioned load-bearing structures or even rejection of thermoplastic composites in the design process.

The shape of the long-term predictions is in line with earlier findings on creep with physical ageing taken into account (e.g. Hastie 1991; Sullivan et al. 1993; Bradshaw and Brinson 1997; Brinson and Brinson 2015) and is influenced by the chosen data analysis method to 
construct the momentary master curves. Although the difference between the momentary master curves seems to be small, clear distinctive long-term creep curves appear. The deviation in compliance at 20 years between the HS and TCM long-term prediction is around $14 \%$. Therefore, the validity of the obtained momentary master curve is important, which emphasises the care that should be taken to minimise for example fluctuations in humidity, temperature and stress and differences in fibre-volume fraction in experimental testing. One could argue that the HS master curve is not valid due to the non-smooth master curve and the poor conformity of the shift factors with the Arrhenius equation (10). Calculation of the root mean square (RMS) error between the measured creep response at $50^{\circ} \mathrm{C}$ and the HS, DS and TCM master curves results in 8.2e-11, 6.1e-13 and 5.3e-13 $\mathrm{Pa}^{-1}$, respectively, which increases the uncertainty about the validity of the HS master curve. Neglecting the HS longterm prediction narrows the long-term compliance range, as the DS and TCM long-term curve deviate $4.2 \%$ with respect to the DS compliance value at 20 years. Further research to experimentally validate the momentary master curve construction and ETT application is strongly recommended.

\section{Conclusions}

Bias extension creep tests were performed on carbon woven fabric reinforced poly(phenylene sulphide) (C/PPS) to characterise the physical ageing process by means of the ageing shift rate conform the approach outlined by Struik (1977) with only horizontal shifting, which indicates thermo-rheologically simple material behaviour. The non-aged (momentary) in-plane shear creep compliance curves, measured at different temperatures below the glass transition temperature, did not superimpose correctly using the ordinary timetemperature superposition principle (TTSP). Therefore, three alternative approaches were attempted to resolve this issue, namely the omission of start-up data, a double (horizontal and vertical) shift and a thermo-rheologically complex material (TCM) shift proposed by Urzhumtsev (1974), to obtain smooth momentary master curves. The validity of the first approach was still questionable due to the imperfect smoothness and the relative poor conformity of the shift factors with the kinetic rate theory. On the contrary, both the double shift and the TCM shift resulted in a smooth master curve with shift factors that were in better agreement with theory. The vertical scaling in the double shift, however, lacks a physical foundation and cropped the resulting master curve on the logarithmic time scale, which was not the case for the TCM master curve.

The effective time theory developed by Struik (1977) was used to correct the obtained momentary master curves by means of the ageing shift rate, which resulted in a lower evolving creep response over time compared with the momentary response. Consequently, a longterm prediction of the in-plane shear creep compliance of C/PPS under the influence of physical ageing was obtained. The momentary compliance prediction increased progressively over time, whereas the physically aged creep prediction tends to increase towards a finite value, which is crucial for secure long-term operations of load-bearing structures using thermoplastic composite materials.

Acknowledgements The authors would like to acknowledge TPRC for supplying the material used. Further, the help of the lab technicians, Bert Vos, Nick Helthuis and Dries van Swaaij, was highly appreciated. Finally, the authors would like to thank Leon Govaert and Laurent Warnet for the valuable discussions.

This research did not receive any specific grant from funding agencies in the public, commercial, or not-for-profit sectors. 
Publisher's Note Springer Nature remains neutral with regard to jurisdictional claims in published maps and institutional affiliations.

Open Access This article is distributed under the terms of the Creative Commons Attribution 4.0 International License (http://creativecommons.org/licenses/by/4.0/), which permits unrestricted use, distribution, and reproduction in any medium, provided you give appropriate credit to the original author(s) and the source, provide a link to the Creative Commons license, and indicate if changes were made.

\section{Appendix: Thermo-rheologically complex material shift}

Urzhumtsev (1974) and Gol'dman et al. (1977) studied on a temperature and time-dependent shift factor, $a_{T}(T, t)$, which enables a dedicated shift of the spectrum of retardation times in order to take thermo-rheologically complex material (TCM) behaviour into account. The proposed method will be outlined briefly.

First, assume that the momentary creep compliance at temperature $T$, and reference temperature $T_{0}$, can be written as,

$$
\begin{aligned}
S_{T}(\log t) & =\left|\int \frac{\partial S_{T}(\log t)}{\partial \log t} \mathrm{~d} \log t\right|_{T} ; \\
S_{T_{0}}\left(\log \left[t a_{T}\right]\right) & =\left|\int \frac{\partial S_{T_{0}}\left(\log \left[t a_{T}\right]\right)}{\partial \log \left(t a_{T}\right)} \mathrm{d} \log \left(t a_{T}\right)\right|_{T_{0}},
\end{aligned}
$$

in which $a_{T}$ depends on temperature and time; $a_{T}(T, t)$. A correct horizontal shift should result in equal compliance values,

$$
\begin{aligned}
& \left|\int \frac{\partial S_{T}(\log t)}{\partial \log t} \mathrm{~d} \log t\right|_{T} \\
& \quad=\left|\int \frac{\partial S_{T_{0}}\left(\log \left[t a_{T}\right]\right)}{\partial \log \left(t a_{T}\right)} \mathrm{d} \log \left(t a_{T}\right)\right|_{T_{0}} .
\end{aligned}
$$

By differentiating both sides with respect to $\log t$ and after rewriting one obtains,

$$
\frac{\partial \log a_{T}(T, t)}{\partial \log t}=\frac{\left|\frac{\partial S_{T}(\log t)}{\partial \log t}\right|_{T}}{\left|\frac{\partial S_{T_{0}}\left(\log \left[t a_{T}(T, t)\right]\right)}{\partial \log \left[t a_{T}(T, t)\right]}\right|_{T_{0}}}-1=f(T, \log t),
$$

in which the left hand side is equal to zero in the case of a TSM, which was also shown by Fesko and Tschoegl (1971). Hence, the shape of the creep curves is conserved, as previously mentioned. In order to obtain an expression for the temperature and time-dependent shift factor, Eq. (A.3) needs to be integrated. Urzhumtsev (1974) carried out this integration,

$$
\begin{aligned}
\log a_{T}(T, t) & =\left|\int_{-\infty}^{\log t} f(T, \log t) \mathrm{d} \log t\right|_{T-T_{0}} \\
& =\left|\int_{-\infty}^{\log t_{0}} f(T, \log t) \mathrm{d} \log t\right|_{T-T_{0}}+\left|\int_{\log t_{0}}^{\log t} f(T, \log t) \mathrm{d} \log t\right|_{T-T_{0}} \\
& \left.=\log a_{T}\left[\left(T-T_{0}\right), t_{0}\right)\right]+\log a_{T}\left[\left(T-T_{0}\right), \theta\right]
\end{aligned}
$$




$$
=\log a_{T}\left(T, t_{0}\right)\left[1+\frac{\log a_{T}(T, \theta)}{\log a_{T}\left(T, t_{0}\right)}\right]
$$

where $t_{0}$ is a reference time and $\theta=\log t-\log t_{0}$. The first term of the final result depends only on temperature. The second term still depends on temperature and time. Hence, an adequate function, $f(T, \theta)$, to characterise $\log a_{T}(T, \theta)$ needs to be found. It would be convenient if $f(T, \theta)$ could be expressed independent of temperature. Therefore, a similarity function was introduced by Urzhumtsev (1974),

$$
\frac{\partial f}{\partial \theta}=\frac{1}{\log a_{T}\left(T, t_{0}\right)} \frac{\partial \log a_{T}(T, \theta)}{\partial \theta},
$$

which was approximated by a sum of exponentials,

$$
\frac{\partial f}{\partial \theta}=\sum_{i=1}^{n} \frac{C_{i}}{b_{i}} e^{-\theta / b_{i}} .
$$

Hence,

$$
f(\theta)=\frac{C}{n} \sum_{i=1}^{n}\left(1-e^{-\theta / b_{i}}\right) \quad \text { with } C=\sum_{i=1}^{n} C_{i},
$$

which can be used to approximate the last term in Eq. (A.4),

$$
\log a_{T}(T, t)=\log a_{T}\left(T, t_{0}\right)\left[1+\frac{C}{n} \sum_{i=1}^{n}\left(1-e^{-\theta / b_{i}}\right)\right] .
$$

The parameters $\left(C, b_{i}\right)$ were found empirically. Urzhumtsev (1974) checked the validity of the outlined procedure with a long-term control experiment, which resulted in a satisfactory agreement. Note that the used shift factor, $a_{T}$, is positive for a shift to the right. In the past, this was the convention in Russian literature (Griffith 1980), which, however, has no consequences for the final result.

\section{References}

Airbus S.A.S., A350 XWB family, accessed on 2018-11-16 (2018). https://www.airbus.com/aircraft/ passenger-aircraft/a350xwb-family.html

Albouy, W., Vieille, B., Taleb, L.: Investigations on the creep/recovery behavior of woven-ply carbon fiber reinforced PPS over glass transition temperature. In: Proc. of 15th Eur. Conference on Compos. Mater. (ECCM 2012), Venice, Italy (2012)

Alwis, K.G.N.C., Burgoyne, C.J.: Time-temperature superposition to determine the stress-rupture of aramid fibres. Appl. Compos. Mater. 13(4), 249-264 (2006)

Amiri, A., Yu, A., Webster, D., Ulven, C.: Bio-based resin reinforced with flax fiber as thermorheologically complex materials. Polymers 8(4), 153 (2016)

Barbero, E.J.: Prediction of long-term creep of composites from doubly-shifted polymer creep data. J. Compos. Mater. 43(19), 2109-2124 (2009)

Barbero, E.J.: Time-temperature-age superposition principle for predicting long-term response of linear viscoelastic materials. In: Guedes, R. (ed.) Creep and Fatigue in Polymer Matrix Composites, vol. 2010, pp. 48-69. Woodhead Publishing Ltd., Cambridge (2010). Chap. 2

Bradshaw, R.D., Brinson, L.C.: Physical aging in polymers and polymer composites: an analysis and method for time-aging time superposition. Polym. Eng. Sci. 37(1), 31-44 (1997)

Brinson, H.F., Brinson, L.C.: Polymer Engineering Science and Viscoelasticity: An Introduction, 2nd edn. Springer, New York (2015) 
Brinson, L.C., Gates, T.S.: Effects of physical aging on long term creep of polymers and polymer matrix composites. Int. J. Solids Struct. 32(6-7), 827-846 (1995)

Carnevale, P.: Fibre-matrix interfaces in thermoplastic composites. Ph.D. thesis, Delft University of Technology, Delft, the Netherlands (2004)

Cerrada, M.L., McKenna, G.B.: Physical aging of amorphous PEN: isothermal, isochronal and isostructural results. Macromolecules 33(8), 3065-3076 (2000)

Fesko, D.G., Tschoegl, N.W.: Time-temperature superposition in thermorheologically complex materials. J. Polym. Sci., C Polym. Symp. 35(1), 51-69 (1971)

Findley, W.N., Lai, J.S., Onaran, K.: Creep and Relaxation of Nonlinear Viscoelastic Materials with an Introduction to Linear Viscoelasticity. North-Holland, Amsterdam (1976)

Flory, A., McKenna, G.B.: Finite step rate corrections in stress relaxation experiments: a comparison of two methods. Mech. Time-Depend. Mater. 8(1), 17-37 (2004)

Gates, T.S., Feldman, M.: Time dependent behavior of a graphite/thermoplastic composite and the effects of stress and physical aging. In: 34th AIAA/ASME/ASCE/AHS/ASC DSM Conference, La Jolla (CA), USA. NASA Langley Research Center, Hampton (1993)

Gol'dman, A.Y., Murzakhanov, G.K., Soshina, O.A.: Temperature-time analogy applied to thermorheologically complex polymeric materials: 1 . Mixtures of partially crystalline polymers with elastomers. Polym. Mech. 13(4), 516-522 (1977)

Gol'dman, A.Y., Murzakhanov, G.K., Soshina, O.A.: Temperature-time analogy applied to thermorheologically complex polymer materials: 2 . Mixtures of thermoplastic and thermosetting polymers. Polym. Mech. 14(3), 348-352 (1978)

Griffith, W.I.: The accelerated characterization of viscoelastic composite materials. Ph.D. thesis, Virginia Polytechnic Institute and State University, Blacksburg (VA), United States of America (1980)

Guo, Y., Bradshaw, R.D.: Isothermal physical aging characterization of polyether-ether-ketone (PEEK) and polyphenylene sulfide (PPS) films by creep and stress relaxation. Mech. Time-Depend. Mater. 11(1), 61-89 (2007)

Guo, Y., Bradshaw, R.D.: Long-term creep of polyphenylene sulfide (PPS) subjected to complex thermal histories: the effects of nonisothermal physical aging. Polymer 50(16), 4048-4055 (2009)

Guo, Y., Wang, N., Bradshaw, R.D., Brinson, L.C.: Modeling mechanical aging shift factors in glassy polymers during nonisothermal physical aging. I. Experiments and KAHR- $a_{\text {te }}$ model prediction. J. Polym. Sci., Part B, Polym. Phys. 47(3), 340-352 (2009)

Hastie, R.L.: The effect of physical aging on the creep response of a thermoplastic composite. Ph.D. thesis, Virginia Polytechnic Institute and State University, Blacksburg (VA), United States of America (1991)

Hung, K.C., Wu, J.H.: Effect of $\mathrm{SiO}_{2}$ content on the extended creep behavior of $\mathrm{SiO}_{2}$-based wood-inorganic composites derived via the sol-gel process using the stepped isostress method. Polymers 10(4), 409 (2018)

Hutchinson, J.M.: Physical aging in polymers. Prog. Polym. Sci. 20(4), 703-760 (1995)

Kê, T.S.: Experimental evidence of viscous behaviour of grain boundaries in metals. Phys. Rev. 71(8), 533546 (1947)

Kovacs, A.J., Aklonis, J.J., Hutchinson, J.M., Ramos, A.R.: Isobaric volume and enthalpy recovery of glasses. II. A transparent multiparameter theory. J. Polym. Sci., Part B, Polym. Phys. 17(7), 1097-1162 (1979)

Lai, J., Bakker, A.: Analysis of the non-linear creep of high-density polyethylene. Polymer 36(1), 93-99 (1995)

Leaderman, H.: Elastic and creep properties of filamentous materials. Ph.D. thesis, Massachusetts Institute of Technology, Cambridge (MA), United States of America (1941)

Lee, S., Knauss, W.G.: A note on the determination of relaxation and creep data from ramp tests. Mech. Time-Depend. Mater. 4(1), 1-7 (2000)

Luinge, J.W.: Toray TCAC, personal communication (2018)

McCrum, N.G., Morris, E.L.: Lamellar boundary slip in polyethylene. Proc. R. Soc. Lond. Ser. A, Math. Phys. Sci. 292(1431), 506-529 (1966)

McKenna, G.B.: Mechanical rejuvenation in polymer glasses: fact or fallacy? J. Phys. Condens. Matter 15(11), 737-760 (2003)

Motta Dias, M.H., Jansen, K.M.B., Luinge, H., Nayak, K., Bersee, H.E.N.: The influence of fiber-matrix adhesion on the linear viscoelastic creep behavior of CF/PPS composites. In: 1st Int. Conference on Ageing Mater. Struct, Delft, the Netherlands: Delft University of Technology (2014)

Motta Dias, M.H., Jansen, K.M.B., Luinge, J.W., Bersee, H.E.N., Benedictus, R.: Effect of fiber-matrix adhesion on the creep behavior of CF/PPS composites: temperature and physical aging characterization. Mech. Time-Depend. Mater. 20(2), 245-262 (2016)

Muliana, A., Nair, A., Khan, K.A., Wagner, S.: Characterization of thermo-mechanical and long-term behaviors of multi-layered composite materials. Compos. Sci. Technol. 66(15), 2907-2924 (2006) 
Schwarzl, F., Staverman, A.J.: Time-temperature dependence of linear viscoelastic behavior. J. Appl. Phys. 23(8), 838-843 (1971)

Smith, O.: Revealed: the oldest passenger planes still in service, The Telegraph (2017), accessed on 2018-1116. https://www.telegraph.co.uk/travel/travel-truths/oldest-passenger-plane-still-in-service/

Spruiell, J.E., Janke, C.J.: A review of the measurement and development of crystallinity and its relation to properties in neat poly(phenylene sulfide) and its fiber reinforced composites. Tech. rep., Oak Ridge National Laboratory, Oak Ridge (TN), United States of America (2004)

Struik, L.C.E.: Physical aging in amorphous polymers and other materials. Ph.D. thesis, Technische Hogeschool Delft, Delft, the Netherlands (1977)

Sullivan, J.L., Blais, E.J., Houston, D.: Physical aging in the creep behavior of thermosetting and thermoplastic material. Compos. Sci. Technol. 47(4), 389-403 (1993)

Tschoegl, N.W., Knauss, W.G., Emri, I.: Poisson's ratio in linear viscoelasticity-a critical review. Mech. Time-Depend. Mater. 6(1), 3-51 (2001)

Urzhumtsev, Y.S.: Time-temperature superposition for thermorheologically complex materials. Polym. Mech. 10(2), 180-185 (1974)

Vieille, B., Taleb, L.: About the influence of temperature and matrix ductility on the behavior of carbon woven-ply PPS or epoxy laminates: notched and unnotched laminates. Compos. Sci. Technol. 71(7), 998-1007 (2011)

Vieille, B., Aucher, J., Taleb, L.: Comparative study on the behavior of woven-ply reinforced thermoplastic or thermosetting laminates under severe environmental conditions. Mater. Des. 35(1), 707-719 (2012)

Wisnom, M.R.: The effect of fibre rotation in $\pm 45^{\circ}$ tension tests on measured shear properties. Composites 26(1), 25-32 (1995)

Zapas, L.J., Crissman, J.M.: Creep and recovery behaviour of ultra-high molecular weight polyethylene in the region of small uniaxial deformations. Polymer 25(1), 57-62 (1984) 